\title{
MAXIMIZING THE UTILIZATION OF OPERATING ROOMS WITH STOCHASTIC TIMES USING SIMULATION
}

\author{
Jean-Paul M. Arnaout \\ Sevag Kulbashian \\ Department of Industrial Engineering \\ Lebanese American University \\ Byblos, LEBANON
}

\begin{abstract}
This paper addresses a surgery rooms scheduling problem. The problem is modeled as a parallel machine scheduling problem with sequence dependent setup times and an objective of minimizing the makespan. This is a NPhard problem and in this paper, a solution heuristic is developed and compared to existing ones using simulation. The results and analysis obtained from the computational experiments proved the superiority of the proposed algorithm LEPST over the other algorithms presented.
\end{abstract}

\section{INTRODUCTION AND BACKGROUND}

Now more than ever, the healthcare industry needs to respond to the swell in challenges and pressures in its environment. The constant increase in number of patients and surgeries require new unconventional methods that aim at increasing the efficiency and particularly improving the Operating Room (OR) utilization. The latter is one of the largest revenue and cost centers in the healthcare industry (Tsoy et al. 2004); i.e. it has a great potential of reducing expenses, especially that it affects many parts in the hospital such as resource management, financial management, purchasing, and many other areas along with the patients' waiting times.

The current norm in hospitals is that patients wait long hours before they are checked in and prepared for surgery. Unsuccessful scheduling of ORs is simultaneously decreasing the rooms' utilization and increasing the patients' delays, an act that is very costly. Operations managers' main target is to attain more accurate scheduling techniques in order to improve the ORs utilization.

Following the above, this paper addresses the problem of operating rooms' scheduling (non emergency cases) inside a local hospital with an objective of maximizing the utilization of the rooms using a dispatching rule. A dispatching rule is a priority rule, based on which the operations are organized in the different rooms. Currently, most hospitals including the one in this study assign block times to surgeons' groups based on their requests. Then, surgeons contact the administrative office for a specific starting time for surgery and request a reservation within allocated block time. The reservation within a certain time block is done on "first come first served" basis.

In our local hospital, the operations that are non emergency cases are scheduled and planned on a daily basis. There is a special room for the emergency cases, which does not affect the OR scheduling. The hospital has four operating rooms, and eleven different major types of operations are executed in those rooms. Each operation type has a certain stochastic duration, and also a preparation phase before the operation. The duration of the preparation is affected by the sequence of the operations in a particular OR. This scheduling problem when translated to machine scheduling becomes the scheduling of jobs with sequence dependent setup times on identical parallel machines with the objective of minimizing the maximum completion time. This is at least a NP-hard problem as the simplified problem of two identical machines with no setup times is NP-hard in the ordinary sense (Bruno, Downey, and Frederickson 1981). Discrete event simulation will be used to model and test three heuristics for the problem addressed in this paper.

The rest of this paper is organized as follows. In section 2, the related research is summarized. In section 3, the problem statement and objective function are presented. Section 4 contains description of the heuristics developed and used. The simulation model verification and validation are presented in section 5; the computational results and output analysis are described in section 6. Finally, we conclude our results in section 7.

\section{RELATED RESEARCH}

The maximization of the OR utilization has been the aim of several researchers. Few of them used simulation as a tool to model and solve healthcare scheduling problems. 


\section{Arnaout and Kulbashian}

Shmitz and Kwak (1972) developed a Monte Carlo simulation model for OR and recovery room usage. The authors used their model to determine the improvements if bed capacity is increased, and the surgical procedures' time and space requirements for both the operating and recovery rooms. Dexter et al. (1999) used computer simulation for scheduling operating rooms' Block Time in order to improve utilization; no preparation time was considered in their paper. Dexter (2000) analyzed the effects of scheduling on operating rooms' labor costs, and the effect of having a high OR utilization on the salaries of the personnel. Moreover, the author addressed some operational goals. Tsoy et al. (2004) introduced a genetic algorithm approach for the surgery operating rooms scheduling problem. The authors assumed the operations times to be deterministic. Jebali, Alouane, and Ladet (2006) introduced a two-step approach for operating room scheduling, where operations are assigned to ORs in the first step, and then these operations are sequenced in the second step. The authors considered all times to be deterministic. For more readings on techniques to construct the operating room schedule, the reader may refer to (Guinet and Chaabane 2003; Kharraja, Chaabane, and Marcon 2002).

The most common objectives studied in parallel machine scheduling are minimization of completion time, tardiness, and makespan. The identical parallel machine problem with the objective of minimizing the makespan has been proven to be a NP problem (Sethi 1977; Garey and Johnson 1979). Due to this complexity, it became a common and acceptable practice to find suitable heuristics instead of optimal solutions for such problems. Following the conventional scheduling notation, the identical parallel machine problem can be denoted by $\mathrm{P}_{\mathrm{m}} / / \mathrm{C}_{\max }$, where $m$ refers to the number of machines, and $\mathrm{C}_{\max }$ to the maximum completion time (makespan). In this problem (without setup times) with jobs having a deterministic processing time, the LPT (largest processing time) heuristic gives near optimal results (Graham 1969; Pinedo 1995). As for the same problem but with jobs having a stochastic processing time, the LEPT (largest expected processing time) rule minimizes the expected makespan in the class of nonpreemptive jobs (Pinedo 1995). Lee and Pinedo (1997) addressed the same problem with setup times where all times are deterministic and presented a three phase heuristic for minimizing the sum of the weighted tardiness. Monma and Potts (1989) addressed the problem of two identical parallel machines with batch setup times and claimed that pseudo polynomial algorithms exist for minimizing the makespan when the number of batches is fixed. Min and Cheng (1999) introduced a genetic algorithm for the same objective but without setup times and where the processing times are deterministic.

Stochastic machine scheduling problems have been considered, among others, by Glazebrook (1979), Weiss and Pinedo (1980), Bruno, Downey, and Frederickson (1981), Weber, Varaiya, Walrand (1986), Weiss (1992), Mohring, Shulz, and Uetz (1999), Javidi, Song, and Teneketzis (2001), and Arnaout, Rabadi, and Mun (2006). However, and up to our knowledge, no previous research has addressed the generation of schedules in identical parallel machines with stochastic processing and sequence dependent setup times, and this is where the contribution of this paper lies.

\section{PROBLEM STATEMENT}

The main objective of this research is to find a dispatching rule to schedule the operations in the ORs in a way that maximizes the utilization of the rooms.

\subsection{The OR Scheduling Problem}

Scheduling is present in many areas of the hospital, one of which is the OR. The assignment of daily operations affects several parts of the hospital, e.g. resource management, financial management, and patients waiting times. As the salaries of the staff accounts for most OR costs, it is of great importance that the number of staff remains at its critical needed value, because in the OR scheduling case, the best way to maximize the personnel utilization is to maximize the OR utilization (Dexter et al. 1999).

The OR scheduling problem is translated to machine scheduling as follows:

- The operating rooms are regarded as machines.

- The operations are regarded as jobs.

- Operations can be performed in any room, as on any machine.

- Operations can be performed at any time which is translated as job independence.

- Operations need a preparation of equipment and personnel before they start, this translates to that jobs have a sequence dependent setup time.

- The objective is to organize operations in a way to complete all the operations as soon as possible, by minimizing the maximum completion time at the end of the working day. This can be translated to the minimization of the maximum completion time $\mathrm{C}_{\max }$.

In the case of this paper, we have chosen the scheduling of the operating rooms inside a local hospital in Lebanon. In table 1, a sample schedule of daily operations is given. Figure 1 presents the OR problem as a machine scheduling representation, where each block is an operation (job) and the dotted line divides between the preparation (setup) and the processing times. Note that the scheduling of the operations is done on a daily basis.

Table 1: An example of four ORs scheduling 

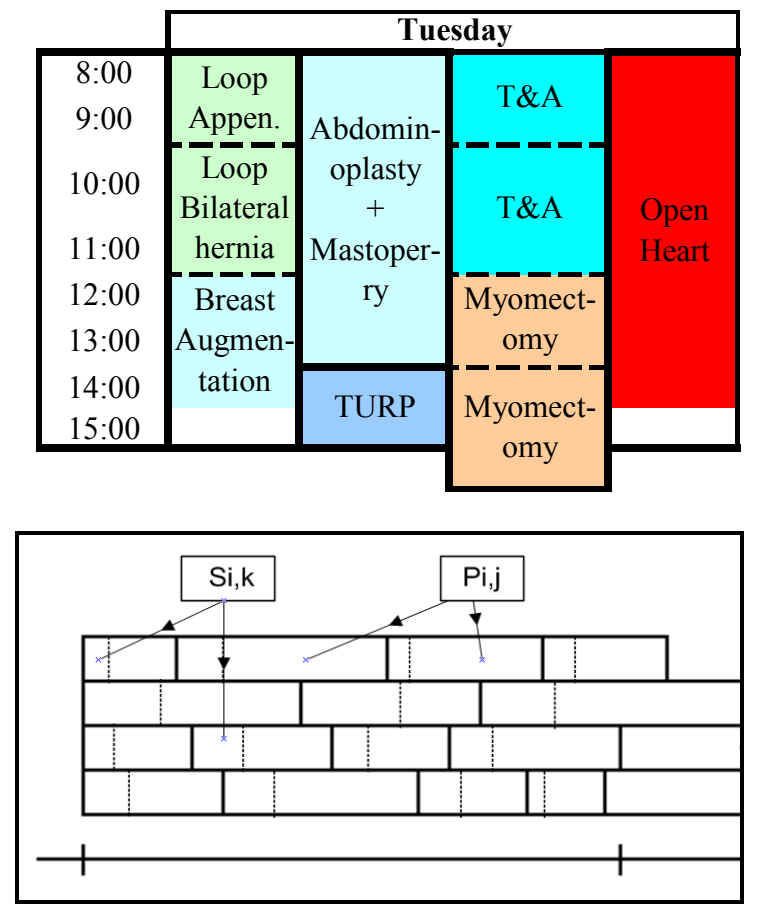

Figure 1: Representation of a 4 machine scheduling model with sequence dependent setup times (Fredi et al. 2001).

Some of the constraints/assumptions for this problem are listed as follows. First, the operations are considered independent from one another; the operating rooms are identical and parallel meaning any operation can be scheduled on any OR at any time; the scheduling of operations is a daily process, the quantity of operations scheduled is based on the daily capacity, and all the operations are scheduled before the day begins.

The problem statement in machine scheduling terms is the following:

Where,

$$
\mathrm{P}_{\mathrm{m}} / \mathrm{S}_{\mathrm{ik}} / \mathrm{C}_{\max }
$$

$\mathrm{P}_{\mathrm{m}}$ : $\mathrm{m}$ parallel identical machines.

$\mathrm{S}_{\mathrm{ik}}$ : The jobs are constrained with setup times.

$\mathrm{C}_{\max }$ : The objective is to minimize $\mathrm{C}_{\max }$ (maximum completion time).

\subsection{Problem Constraints}

There are $\mathrm{m}$ identical parallel machines, and $\mathrm{n}$ independent jobs to be scheduled on the machines. Each machine is able to process any type of jobs and a job has the same processing time on any machine (Identical). The jobs are simultaneously available at the beginning of the scheduling horizon (at time zero). Further more, each job can be processed on any of the machines but needs to be processed by one machine only, and each machine is capable of processing only one job at a time. Job preemption is not allowed and there is no processing precedence on any of the machines. The machine setup times are dependent on jobs' sequence where setup times depend on both the job just completed and the next job to be processed. Setup times are assumed to be machine independent such that regardless to which machine jobs $k$ and $i$ are assigned, $s_{k i}$ would be the setup time required if job $i$ is scheduled after job $k$.

Our main objective is to have the maximum utilization of the operating rooms. The makespan, defined as $\max \left(\mathrm{C}_{1}, \mathrm{C}_{2} \ldots \ldots, \mathrm{C}_{\mathrm{n}}\right)$, is equivalent to the completion time of the last job to leave the system. A minimum makespan usually implies a high utilization of the machines (Pinedo 1995). Hence, by minimizing $\mathrm{C}_{\max }$, we maximize the utilization of the operating rooms.

\section{HEURISTIC ALGORITHMS}

In the following sections, different heuristics are presented and compared in order to determine the most appropriate one for our problem. The jobs' processing and setup times are stochastic and drawn from different uniform distributions. Whenever a job is called by any algorithm to be sorted with the other jobs or sent to a machine, it will be assigned a processing time and setup time following some uniform distribution; this is discussed more in section 6 .

\subsection{LPT and SPT}

Longest Processing Time First (LPT) rule gives the priority to the job that has the longest processing time to be scheduled first. Under LPT, the shortest job is the last job to start its processing and also the last job to finish its processing.

Shortest Processing Time First (SPT) gives the priority to the job that has the smallest processing time to be scheduled first. Under SPT, the longest job is the last job to start its processing and also the last job to finish its processing.

During the schedule's execution for the multiple machine case, LPT and SPT send the jobs to the machine that has the minimum completion time.

\subsection{LEPT and SEPT}

LEPT and SEPT are very similar to LPT and SPT, but they differ in that they consider the average processing times of jobs. In other words, the advantages over the LPT and SPT are the ability to schedule jobs in stochastic environments (Pinedo 1995).

In order to use the LEPT or SEPT, we must calculate the averages of the expressions of processing times. In particular, whenever we want to schedule a job on the machines, LEPT will pick from the unscheduled jobs the one with the maximum $E_{\mathrm{i}}$, while SEPT will pick the job with 


\section{Arnaout and Kulbashian}

the minimum $\mathrm{EP}_{\mathrm{i}}$; the chosen job using either rule will be sent to the machine with the smallest completion time. Note that $\mathrm{EP}_{\mathbf{i}}$ is the expected processing time of job $i$ on any machine for $i=1, \ldots, \mathrm{n}$.

The limitation of LEPT and SEPT for the OR problem is that they do not account for setup (preparation) times.

\subsection{Longest Expected Processing with Setup Time (LEPST)}

The LEPST is the dispatching rule that is introduced for OR scheduling in this study; it is an extension to LEPT where the setup times are considered. The main challenge of the problem is the consideration of the sequence dependent setup time. It is shown that the $\mathrm{P}_{\mathrm{m}} / \mathrm{S}_{\mathrm{j}, \mathrm{k}} / \mathrm{C}_{\mathrm{max}}$ problem is strongly NP-hard (Pinedo 1995).

Let $S$ be a set containing the unscheduled jobs.

1. Find job $i$ and machine $j$ where (1) is at its maximum:

$$
\left[\mathrm{C}_{\mathrm{kj}}+\left(\mathrm{EP}_{\mathrm{i}} * \alpha\right)+\operatorname{Max}\left(\mathrm{S}_{\mathrm{j}, \mathrm{k}, \mathrm{i}}\right)\right]
$$

where $i \in S$ (index of unscheduled jobs), $j$ is the machine index, and $k$ is the previous job on that specific machine $M j$. $C_{k j}$ refers to the completion time of the last job on machine $j$, and the control parameter $\alpha$ value was determined from Figure 2.

2. After finding both $i$ and $j$, assign job $i$ to machine $M j$, and remove job $i$ from list $S$.

3. If $S=\varnothing$, STOP; else go to Step 1 .

In LEPST, by giving the priority to jobs that have the maximum setup time, there will be less jobs having high setup times assigned at the end of the schedule; hence affecting less the $\mathrm{C}_{\max }$.

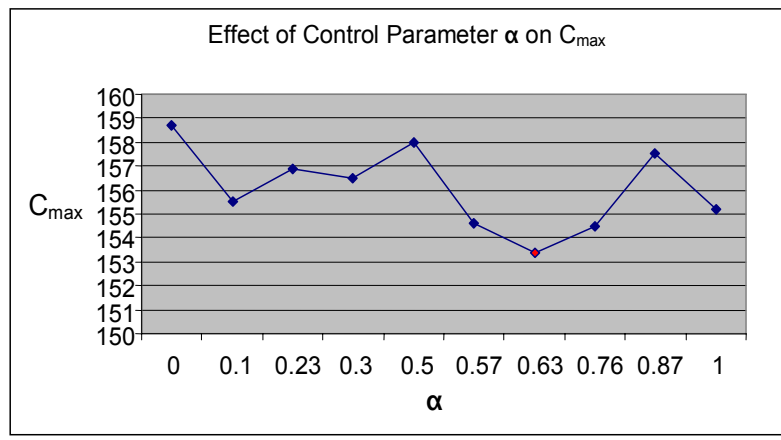

Figure 2: Control Parameter $\alpha$

The chart in Figure 2 describes how the maximum completion time of jobs fluctuates when $\alpha$ is changed while applying LEPST to the problem at hand. Note that the values of $\alpha$ are between 0 and 1, where 1 indicates that the processing and setup times will have equal weights when selecting a job, and 0 indicates that only setup time will decide on the job selection. We can conclude from Figure 2 that the algorithm is giving the best solution when $\alpha=0.63$, and this will be the value to be used in the proposed heuristic LEPST.

In order to test the importance of $\alpha$, we compared LEPST to itself when $\alpha$ was set equal to 1; i.e. given both processing and setup times the same weight. One hundred replications were run for both models and a paired ttest was conducted on the output. Output Analyzer from Rockwell Systems was used for the analysis and the result indicated that on a 95\% Confidence Interval, $\alpha=0.63$ will lead to better Cmax that $\alpha=1$ and the difference is statistically significant. The output analyzer report is shown in Figure 3 below.

\section{MODEL VERIFICATION}

Verification is the process of ensuring that the simulation model behaves in the way it was intended according to the modeling assumptions made (Kelton et al. 2004).

Different methods were applied in verifying the behavior of our models:

- We used first deterministic data instead of stochastic data for both the processing and setup times; this allowed us to predict the system's behavior.

- We let only a single entity enters the system, and then followed this entity through all the decisions nodes to ensure that the model's logic is correct.

- We monitored the model's animation, which made it easier to detect any errors in our logic.

- Finally, we put several variable animations, which enabled us to determine which batch number is first scheduled, and which batch is separated.

\section{COMPUTATIONAL TESTS}

The above heuristics have been modeled and compared using the simulation software Arena. The popularity of simulation has been increasing over the past decade mainly due to its ability to deal with very complicated models of correspondingly complicated systems (Kelton, Sadowski, and Sturrock 2004). The reason stochastic data was used for the processing and setup times is to ensure a more real representation of the healthcare environment, where most of the time an operation will not finish on a specific time, but on a range between two times. Simulation is considered to be one of the best approaches to deal with such source of randomness. The jobs' (operations) processing times and machines' setup (preparation) times 


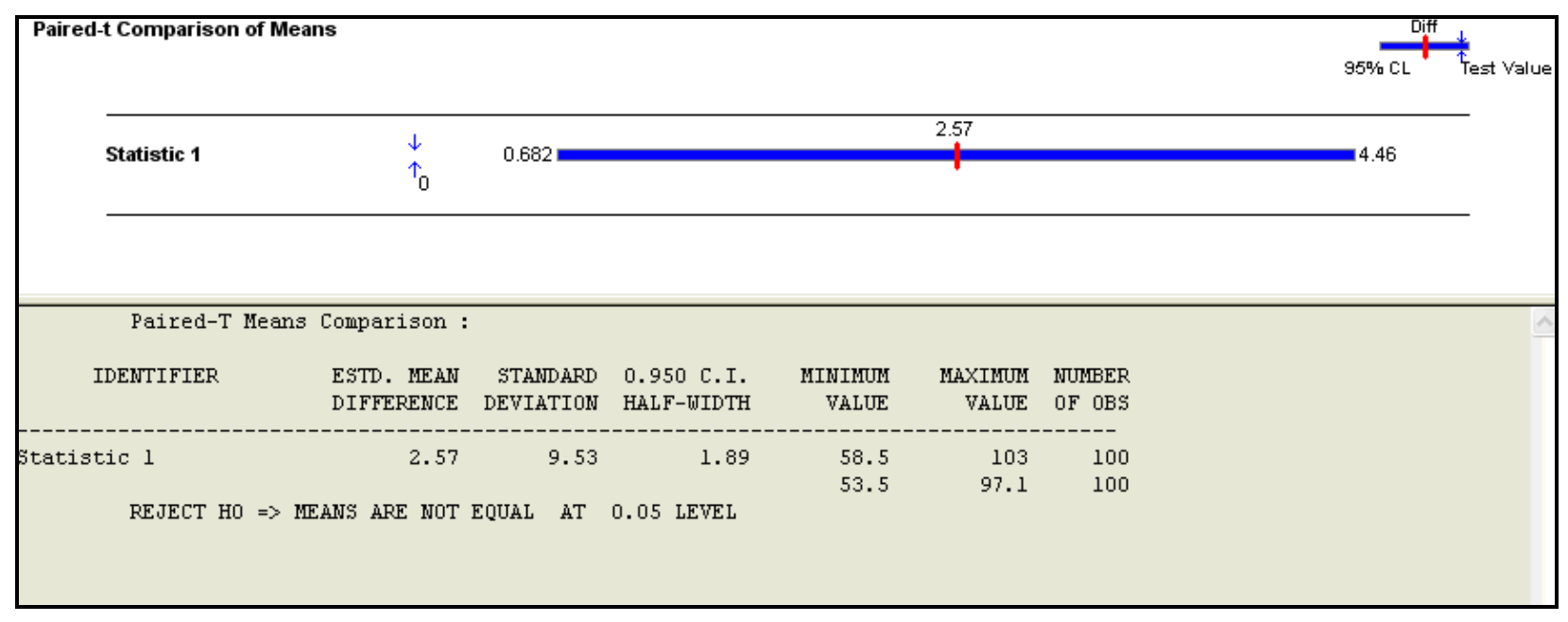

Figure 3: Comparing $\mathrm{Cmax}$ for $\alpha=0.63$ and $\alpha=1$

are stochastic following uniform distributions; these times were drawn from the hospital real data and a sample of operations times is as follows:

Operation type I $\rightarrow \mathrm{U}[1.2,1.5]$

Operation type II $\rightarrow$ U[2.2,2.3]

Operation type III $\rightarrow \mathrm{U}[6.5,6.9]$, etc...

Recall that these values will not be known until the operation is actually being performed in the OR (machine). The reason uniform distributions were used is due to their high variances, ensuring that the presented heuristics are being tested under unfavorable conditions (Weng, $\mathrm{Lu}$, and Ren 2001).

Our simulation model logic is described in Figure 4. Each of the tested heuristics was run for 75 replications (determined through experiments to deliver decent confidence intervals), and paired t-tests were conducted between all the rules (two rules at a time), and a difference between two rules is proven to be statistically significant whenever the p-value was less than 0.05 . These tests proved (for 95\% statistical significance) the following: LEPST performed the best, LEPT second, and SEPT last.

In particular, the results indicated that the utilization of the ORs inside the local hospital in this study can be increased by using the LEPST algorithm with an $\alpha$ of 0.63 . Over a considerable period of time, the rule resulted in a reduction of about 20 minutes per day. The logic behind $\alpha=0.63$ is that there is approximately $60 \%$ weight to the processing time when assigning an operation. This can be understood as the ratio of processing/setup time of the hospital is relatively high.

\section{CONCLUSIONS}

In this paper, we have introduced an effective heuristic algorithm, LEPST, for maximizing the utilization of operating rooms in a local hospital in Lebanon. This problem is equivalent to the minimization of makespan on identical parallel machines with sequence dependent setup times. LEPST was compared to two other algorithms, $L E P T$ and SEPT. All three algorithms were modeled and tested through simulation, and our conclusions were drawn using a large number of replications and several statistical tests. Computational experiments showed that LEPST significantly outperformed the other algorithms.

While the conducted tests show very promising results for OR scheduling, it is worth noting that the rules were compared under static schedules; i.e. operations were available at time 0 of each day. An extension to this work would be to test LEPST (or an expansion of it) under dynamic schedules where operations are arriving over time; such study would be also appropriate for emergency room scheduling.

\section{REFERENCES}

Arnaout, J-P., G. Rabadi, and J.H. Mun. 2006. A Dynamic Heuristic for the Stochastic Unrelated Parallel Machine Scheduling Problem. International Journal of Operations Research 3: 136-143.

Bruno, J.L., P.J Downey, and G.N Frederickson. 1981. Sequencing tasks with exponential service times to minimize the expected flow time or makespan. Journal of the ACM 28: 100-113.

Dexter, F., A. Macario, R Traub, M. Hopwood, and D. Lubarsky. 1999. An operating room scheduling strategy to maximize the use of operating room block time: computer simulation of patient scheduling and survey of patients' preferences for surgical waiting time. Anesthesia \& Analgesia 89: 7-20.

Dexter, F. 2000. A strategy to decide whether to move the last case of the day in an operating room to another empty operating room to decrease overtime labor costs. Anesthesia \& Analgesia 91: 925-928. 
Arnaout and Kulbashian

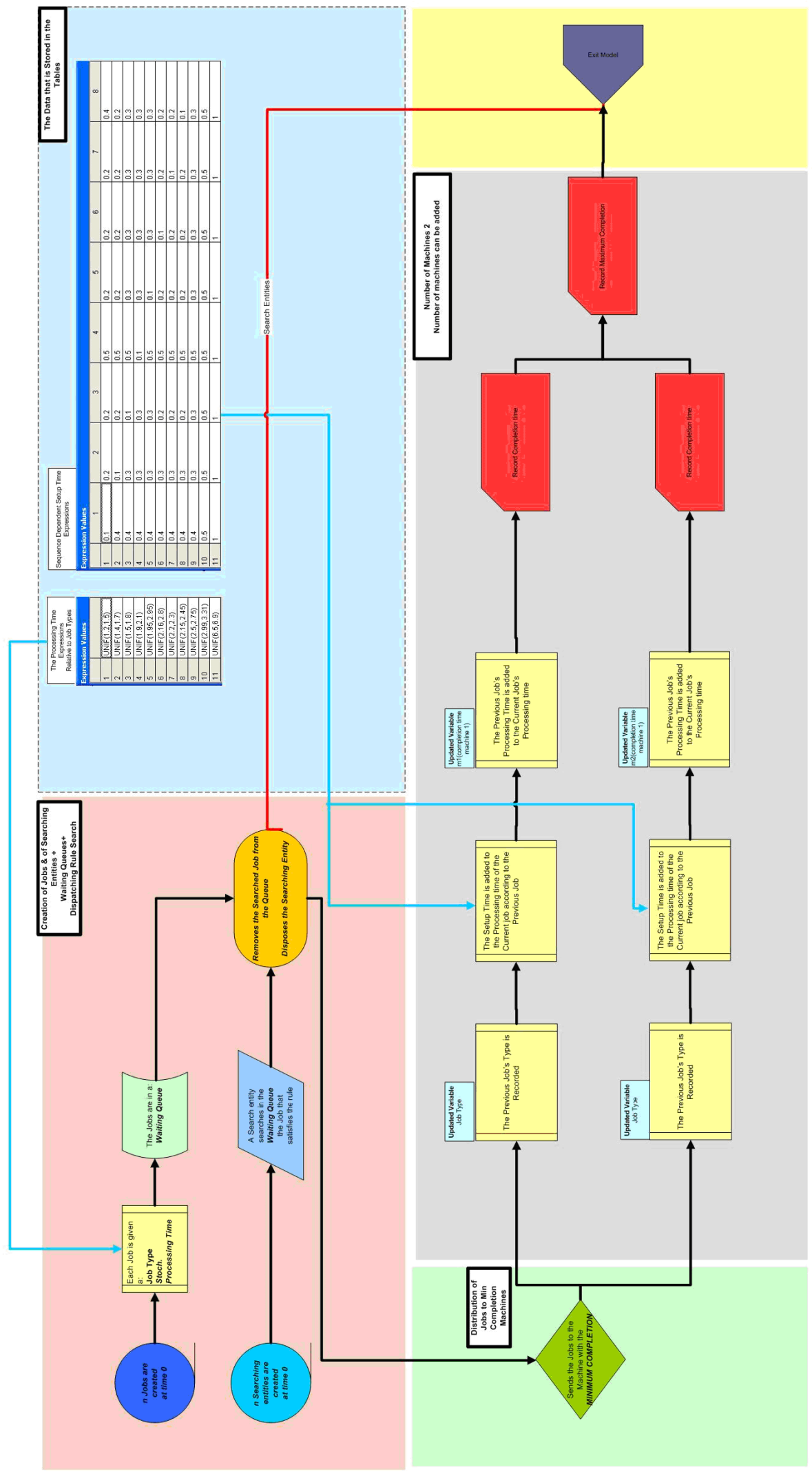

Figure 4: Simulation model flow and Logic 
Garey, MR, and DS Johnson. 1979. Computer and intractability: a guide to the theory of NP-completeness. W H Freeman: San Francisco.

Glazebrook, K.D. 1979. Scheduling tasks with exponential service times on parallel machines. Journal of Applied Probability 16: 685-689.

Graham, R.L. 1969. Boundaries on multiprocessing timing anomalies. SIAM Journal of Applied Mathematics 17: 416-429.

Guinet, A., and S. Chaabane. 2003. Operating theatre planning. International Journal of Production Economics 85: 69-81.

Javidi, T., N-O. Song, and D. Teneketzis. 2001. Expected makespan minimization on identical machines in two interconnected queues. Probability in the Engineering and Informational Sciences 15: 409-443.

Jebali, A., A. Alouane, and P. Ladet. 2006. Operating rooms scheduling. International Journal of Production Economics 99: 52-62.

Kelton, D., R. Sadowski, and D. Sturrock. 2004. Simulation with Arena. $3^{\text {rd }}$ ed. McGraw-Hill Companies, New York.

Kharraja, S, S. Chaabane, and E. Marcon. 2002. Evaluation de performances pour deux stratégies de programmation opératoire de bloc. In: Actes de la $2^{\text {eme }}$ Conf Int Francophone d'Automatique, Nantes, France.

Lee, Y., and M. Pinedo. 1997. Scheduling jobs on parallel machines with sequence-dependent setup times. European Journal of Operational Research 100: 464474.

Min, L., and W. Cheng. 1999. A genetic algorithm for minimizing the makespan in the case of scheduling identical parallel machines. Artificial Intelligence in Engineering 13: 399-403.

Mohring, R., A. Shulz, and M. Uetz. 1999. Approximation in stochastic scheduling: the power of LP-based priority policies. Journal of the ACM (JACM) 46: 924-942.

Monma, CL., and CN. Potts. 1989. On the complexity of scheduling with batch setups. Operations Research 37: 798-804.

Pinedo, M. 1995. Scheduling: Theory, Algorithms, and Systems. Prentice Hall: New Jersey.

Sethi, R. 1977. On the complexity of mean flow time scheduling. Mathematics of Operations Research 2:320-330.

Shmitz, H., and N. Kwak. 1972. Monte Carlo Simulation of Operating-Room and Recovery-Room Usage. $O p$ erations Research 20: 1171-1180.

Tsoy, G., J-P. Arnaout, T. Smith, and G. Rabadi. 2004. A Genetic Algorithm Approach for Surgery Operating Rooms Scheduling Problem. In: Proceedings of the $25^{\text {th }}$ National Conference of the American Society for Engineering Management, Alexandria, Virginia.
Weber, R.R., P. Varaiya, and J. Walrand. 1986. Scheduling jobs with stochastically ordered processing times on parallel machines to minimize expected flowtime. Journal of Applied Probability 23: 841-847.

Weiss, G., and M. Pinedo. 1980. Scheduling tasks with exponential service times on non-identical processors to minimize various cost functions. Journal of Applied Probability 17: 187-202.

Weng, M., J. Lu, and H. Ren. 2001. Unrelated parallel machine scheduling with setup consideration and a total weighted completion time objective. International Journal of Production Economics 70: 215-226.

\section{AUTHOR BIOGRAPHIES}

JEAN-PAUL M. ARNAOUT is an Assistant Professor at the Industrial Engineering Department at the Lebanese American University, Lebanon. He received his $\mathrm{PhD}$ and M.S. from the Department of Engineering Management and Systems engineering at Old Dominion University, Norfolk, Virginia in 2006 and 2003 respectively. He received his bachelor's degree in Mechanical Engineering from the University of Balamand, Lebanon. His Research interests include Optimization Techniques, Modeling and Simulation, and Scheduling and Rescheduling. He can be reached at jparnaoutelau. edu. lb

SEVAG KULBASHIAN is a BE student at the Industrial and Mechanical Engineering Department at the Lebanese American University. His Research interests include Optimization Techniques and Simulation and Modeling. 